African Crop Science Journal by African Crop Science Society is licensed under a Creative Commons Attribution 3.0 Uganda License. Based on a work at www.ajol.info/ and www.bioline.org.br/cs DOI: http://dx.doi.org/10.4314/acsj.v26i2.3

\title{
CONSERVATION DES EXTRAITS AQUEUX DE FEUILLES DE NEEM, D'HYPTIS ET DE PAPAYER ET EFFICACITE CONTRE LES RAVAGEURS DU NIEBE AU BENIN
}

\author{
P.B. AGBOBATINKPO ${ }^{1,3}$, R.H. AHOUANSOU ${ }^{1,4}$, C. AGLI ${ }^{2}$, M. MONTCHO DEDEDJI ${ }^{1}$, \\ B. GNONLONFIN ${ }^{1}$ et B. DOKOUI ${ }^{5}$
}

${ }^{1}$ Programme Technologie Agricole Alimentaire (PTAA), Institut National des Recherches Agricoles du Benin (INRAB), BP 128, République du Bénin

${ }^{2}$ Programme d'Analyse de Politique Agricole (PAPA), Institut National des Recherches Agricoles du Benin (INRAB), Programme Analyse de la Politique Agricole, 01 BP 884 Recette Principale, Cotonou 1, Bénin ${ }^{3}$ Laboratoire de Sciences des Aliments (LSA), Faculté des Sciences Agronomiques (FSA), Université d'Abomey-Calavi (UAC), 03 BP 2819 Jéricho, Cotonou, Bénin

${ }^{4}$ Laboratoire d'Energétique et de Mécanique Appliquée (LEMA), Ecole Polytechnique d'Abomey-Calavi (EPAC), Université d'Abomey-Calavi (UAC), 01 BP: 2009 Cotonou, Benin

${ }^{5}$ Centre de Recherches Agricoles Sud (CRA Sud), Niaouli BP 03 Attogon, Bénin Corresponding author: abpelagie@yahoo.fr, vodabpelagie@gmail.com

(Received 14 February, 2018; accepted 18 April, 2018)

\section{RESUME}

Le niébé (Vigna unguiculata L. Walp) est une des légumineuses largement consommées en Afrique de l'Ouest mais sa culture est sujette aux attaques par les insectes et des maladies. L'utilisation des extraits de plantes naturelles ou de poudre de feuilles végétales est efficace pour le traitement des ravageurs du niébé. Dans cette étude, des tests de stabilisation des extraits aqueux de feuilles telles que le neem (Azadirachta indica A. Juss), le papayer (Carica papaya L.) et l'hyptis (Hyptis suavéolens), obtenus de deux méthodes d'extraction (pilage au mortier + filtration et extracteur motorisé composé de broyeur et de presse) en vue de leur conservation durable ont été réalisés et leur éfficacité testée sur les cultures de niébé. Les extraits ont été stabilisés avec de l'alcool $\left(70{ }^{\circ} \mathrm{C}\right)$, du jus de citron et des huiles essentielles (Ocimum basilicum et Ocimum graticimum). Les genres de champignons Aspergillus spp., Penicillium spp., Fusarium spp., Rhizopus spp. et Nigrospora spp. ont été identifiés au premier jour de l'extraction dans les extraits quelles que soient la feuille et la méthode d'extraction utilisées. Après 1 et 2 mois de conservation des extraits, la plupart des genres de moisissures recensés au début ont été réduits voire absents dans les extraits quelles que soient la feuille et la méthode d'extraction utilisées. Cependant, l'effet inhibiteur des extraits de neem et d'hyptis avec ou sans conservateurs a été moins rapide sur Pénicillium spp. et Rhizopus spp. au cours de la conservation que sur les autres genres identifiés. Les extraits aqueux conservés pendant 45 jours et 6 mois ont été efficaces pour le traitement des plants de niébé par rapport à l'extrait du jour. La conservation des extraits aqueux n'affecte pas leur efficacité pour le traitement des ravageurs du niébé.

Mots Clés: Extracteur, extrait de feuille, stabilisateur, Vigna unguiculata

\begin{abstract}
Cowpea (Vigna unguiculata L. Walp) is one of the leguminous crops, widely consumed in West Africa, but its production is constrained by pests and diseases. The use of natural plant extracts or vegetable leaf powder has been effectively used against cowpea pests. The objective of this study was to evaluate the stability of selected
\end{abstract}


natural plant extracts obtained using two extraction methods (mortar pumping + filtration and motorised extractor composed of grinder and press) and their effectiveness on cowpea crops. The plants included neem (Azadirachta indica A. Juss), papaya (Carica papaya L.) and hyptis (Hyptis suaveolens). The extracts were stabilised with alcohol $\left(70{ }^{\circ} \mathrm{C}\right)$, lemon juice and essential oils (Ocimum basilicum and Ocimum graticimum). Aspergillus spp., Penicillium spp., Fusarium spp., Rhizopus spp. and Nigrospora spp. were identified on the first day of extraction in the leaf extracts regardless of the plant and extraction method. After 1 to 2 months of extract preservation, most fungi initially identified reduced or were absent in the extracts regardless of plant species and method of extraction. However, the inhibitory effect of aqueous extracts with or without conservatives was less for Penicillium spp. and Rhizopus spp. The aqueous extracts were more effective against cowpea pests after 45 days and 6 months of preservation compared to one day extracts. The aqueous leaf extracts can be preserved for a long time without affecting their effectiveness against pests of cowpea.

Key Words: Extractor, leaf extract, stabiliser, Vigna unguiculata L.

\section{INTRODUCTION}

Les crises alimentaires marquées ces dernières années par la famine en Afrique, entraînent le développement des technologies endogènes pour répondre aux besoins alimentaires de la population. La lutte agrobiologique contre les ravageurs des plantes constitue l'un des facteurs de réduction de la famine et différentes technologies ont été mises au point dans le monde (Giglioti et al., 2011, Pazinato et al., 2016), dans la sous-région ouestafricaine (Kwaifai et al., 2015 ; Mamuda et $a l ., 2016$ ) et au Bénin (Affokpon et al., 2017) pour améliorer son efficacité. Des études ont montré l'efficacité des dérivés du neem sur les ravageurs de maïs, du sorgho et du niébé (Mukendi et al., 2014; Tamgno et Ngamo Tinkeu, 2014). Le niébé est la plus importante légumineuse à graines en termes de superficie de production mondiale (14 millions d'hectares) avec une production annuelle de 3,3-5,5 millions de tonnes (FAOSTAT, 2010; CGIAR, 2011; Ouali-N'goran et al., 2014) et l'Afrique produit $64 \%$ de cette production (Makanur et al., 2013; Shanko et al., 2014). La graine du niébé est une importante source de vitamines et de minéraux pour l'alimentation humaine et animale (Hafiz and Damarany, 2006; Dzemo et al., 2010). Différentes études (Munyuli et al., 2008a; Mukendi et al., 2014) ont montré que la culture du niébé rencontre des problèmes d'attaque par les insectes et des maladies. Une perte en rendement de niébé de 80 à $100 \%$ peut être causée par les insectes (Ahmed et al., 2009). Ahmed et al. (2006) ont testé au Nigeria, au Bénin et au Sénégal, l'effet de certaines espèces végétales médicinales ayant des propriétés insecticides et insectifuges sur les insectes phytophages du niébé en champ au stade de floraison et de formation des gousses. Les effets combinés des traitements au SuperDiforce, MaviNPV et au neem, ont permis un contrôle efficace des deux ravageurs (Maruca vitrata et Megalurothrips sjostedti) du niébé au Niger (Kadri et al. (2013).

Ces dix dernières années, le Projet Protection Ecologiquement Durable de Niébé (PEDUNE) devenu Projet Niébé pour l'Afrique (PRONAF) a mis au point et vulgarisé au Bénin une technologie permettant d'utiliser l'effet insecticide et insectifuge d'extraits aqueux de feuilles de neem et de papayer pour le contrôle des ravageurs du niébé. L'étude d'adoption de la technologie de production des extraits aqueux réalisée par le PRONAF en 2001 a montré que le taux d'adoption est de $48 \%$ au Benin, $40 \%$ au Ghana et $35 \%$ au Nigéria (Adeoti et al., 2002). La pénibilité du pilage des feuilles est un des facteurs qui limite cette adoption. Pour lever cette contrainte, l'Institut National de Recherche Agricole du Bénin (INRAB) a mis au point et réalisé un extracteur de solution aqueuse. Les tests en station ont montré que par rapport à la méthode du pilage au mortier, l'extracteur permet de traiter respectivement 3,5 et 7 fois plus rapidement 
les feuilles d'hyptis, de neem et de papayer (Ahouansou et al., 2014). Face à une population africaine estimée à 1,2 milliard en 2016 et au score de l'Indice de la faim en Afrique en 2016 qui demontre que la faim et la sous alimentation sont intolérablemment courante (Grebmer et al., 2016), le défi de la recherche agricole reste énorme. Elle doit accroître la productivité agricole en améliorant les rendements des cultures, la qualité des produits tout en conservant l'environnement (Adéoti et al., 2002). L' augmentation du taux d'adoption des technologies de protection des cultures vivrières à l'aide des extraits aqueux de feuilles s'avère donc nécessaire. Elle réside dans le fait que, pour traiter sa culture, le producteur ait la possibilité de se procurer, tout comme l'engrais chimique et les autres intrants, les extraits aqueux de feuilles dans un point de vente. Cette ambition a été réalisée au Etats-Unis depuis 1999 où les extraits de neem sont utilisés pour fabriquer des insecticides (Bélanger et Musabyimana, 2010). Les travaux de Fandohan et al. (2004) ont montré l'effet des huiles essentielles sur les moisissures se développant sur les cultures vivrières. S'inpirant de ces différents travaux et ceux de Houndété et al. (2002) sur la stabilisation des extraits de feuilles par le jus de citron, la présente étude se propose de mettre au point de méthodes efficaces et durables de conservation des extraits aqueux de feuilles de neem, d'hyptis et de papayer utilisés contre les ravageurs du niébé.

\section{MATERIELS ET METHODES}

Matériel végétal. Les feuilles fraîches de neem, de papayer et d'hyptis ont été utilisées pour produire les extraits aqueux. Les parcelles de niébé ont été traitées avec les extraits obtenus. Les conservateurs utilisés ont été achetés dans le commerce sauf le jus de citron qui a été extrait directement du fruit de citron. Les variétés de niébé utilisées sont la variété Kplobè cultivée dans les communes de
Klouékanmè, d'Aplahoué et de Toffo et la variété Flore cultivée dans la commune d'Adjohoun (Sud Bénin).

Equipements. Le mortier et un complexe extracteur ont été utilisés pour produire les extraits. Le complexe est composé d'un broyeur motorisé pour émietter les feuilles, d'une presse à vis pour extraire le jus après addition de la quantité d'eau nécessaire et d'une capsuleuse. Le complexe est mis au point par le Programme Technologie Agricole Alimentaire (PTAA) de l'INRAB et réalisé par l'atelier BECRREMA de Porto-Novo, Bénin. Un appareil ULV a servi à l'épandage des extraits sur le niébé. Le principe de fonctionnement des équipements est décrit par Ahouansou et al. (2014).

\section{Dispositif expérimental}

Production des extraits et échantillonnage. Deux modes d'extraction ont été réalisés pour chacune des trois feuilles. Les extraits aqueux ont été obtenus à partir de $10 \mathrm{~kg}$ de feuilles fraîches par la méthode traditionnelle et par l'utilisation d'un extracteur. La méthode traditionnelle a consisté à piler les feuilles et à ajouter de l'eau (feuilles / eau : $1 \mathrm{~kg} \mathrm{~L}^{-1}$ ) et un morceau de pain de savon de type « palmida ». L'ensemble est couvert et laissé au repos jusqu'au lendemain. Le mélange est alors filtré à travers un linge fin et est ainsi prèt à être utilisé (PRONAF, 2001).

Test de conservation des extraits aqueux. Les quatre traitements suivants ont été réalisés avec les extraits de neem, de papayer et d'hyptis pour tester leur aptitude à la conservation :

(i) Extrait aqueux par pilage (10 litres) + jus de citron $(30 \mathrm{ml})$;

(ii) Extrait aqueux par pilage (10 litres) + alcool à $70^{\circ} \mathrm{C}(1$ litre $)$; 
(iii) Extrait aqueux par pilage (10 litres) + huile essentielle de Ocimum basilicum $(8 \mathrm{ml})$;

(iv) Extrait aqueux par pilage (10 litres) + huile essentielle de Ocimum gratissimum $(8 \mathrm{ml})$.

L'extrait aqueux sans conservateur est considéré comme essai à blanc. Les mêmes traitements de conservation ont été réalisés avec les extraits aqueux obtenus avec l'extracteur. Les bouteilles brunes (capacité $25 \mathrm{cl}$ ) stérilisées par chauffage à l'eau bouillante pendant environ $20 \mathrm{mn}$ ont été remplies par les extraits et capsulées. Elles ont été stockées et conservées à la température ambiante $\left(28^{\circ} \mathrm{C}+/-2^{\circ} \mathrm{C}\right)$ dans des casiers simplement superposées.

Caractérisation microbiologique des échantillons. Les extraits ont été évalués sur le plan microbiologique par le dénombrement et l'identification des moisissures. Les analyses des extraits ont été réalisées juste à la préparation pour apprécier l'état sanitaire de l'extrait avant la mise en conservation et après un (1) et deux (2) mois de conservation. Les échantillons non embouteillés et sans conservateurs ont été également analysés quatre (4) jours après la production des extraits. Les différents extraits de feuilles (neem, papayer et hyptis) ont été ensemencés sur le milieu de culture PDA (Potato Dextrose Agar). Les boîtes ensemencées ont été incubées à une température de $25^{\circ} \mathrm{C}$ pendant cinq (5) jours ; après quoi les colonies de champignons ont été dénombrées et identifiées selon la méthode décrite par Fandohan et al. (2004).

Efficacité technique des extraits aqueux de neem conservés. Les essais se sont déroulés dans des villages choisis dans chacune des communes de Klouékanmè, d'Aplahoué, de Toffo et d'Adjohoun. Trois producteurs ont été retenus par village. Chaque producteur dispose d'une parcelle semée de niébé. A l'intérieur de la parcelle, sept (7) sousparcelles de $10 \mathrm{~m}$ sur $10 \mathrm{~m}$ ont été délimitées.
Six (6) sous-parcelles représentent les traitements d'extraits aqueux (sans conservateur) de neem, de papayer et d'hyptis obtenus soit avec le mortier, soit avec l'extracteur. La septième sous-parcelle représente le témoin (traitement chimique traditionnel avec le produit Fénitrothion).

Les parcelles des producteurs des villages Toffo-gare, Avassa (commune de Toffo), Davihoué, Gbécochihoué (commune de Klouékanmé) et Kpéta (Commune d'Aplahoué) ont été traitées avec les extraits du jour. $\mathrm{Ce}$ test est conforme à la méthode actuelle qui consiste à traiter les parcelles le jour de l'extraction. Les traitements des extraits aqueux ont été appliqués dès l'apparition des boutons floraux à raison de $150 \mathrm{ml} 100 \mathrm{~m}^{-2}$ soit 15 litres ha $^{-1}$. Les parcelles sont traitées tous les 7 jours. Ainsi, dans chacune de ces localités, les traitements suivants ont été réalisés par producteur :

(i) Parcelle $\mathrm{P}_{0}$ : Traitement avec le produit Fénitrothion (traitement paysan) témoin ;

(ii) Parcelle $\mathrm{P}_{1}$ : Extrait aqueux de feuilles de neem pilées au mortier ;

(iii) Parcelle $\mathrm{P}_{2}$ : Extrait aqueux de feuilles de neem obtenu à l'extracteur;

(iv) Parcelle $\mathrm{P}_{3}$ : Extrait aqueux de feuilles de papayer pilées au mortier ;

(v) Parcelle $\mathrm{P}_{4}$ : Extrait aqueux de feuilles de papayer obtenu à l'extracteur ;

(vi) Parcelle $\mathrm{P}_{5}$ : Extrait aqueux de feuilles de hyptis pilées à la main ;

(viii) Parcelle $\mathrm{P}_{6}$ : Extrait aqueux de feuilles de hyptis obtenu à l'extracteur.

Les parcelles des producteurs de Assouhoué (commune de Klouekanmé) ont été traitées avec les extraits aqueux de neem conservés pendant 45 jours tandis que celles des 
producteurs de Agonguè et de Gangban (commune d'Adjohoun) ont été traitées avec les extraits aqueux de neem conservés pendant six (6) mois. Sur chaque site, les traitements réalisés sont présentés dans le Tableau 1.

Analyses statistiques. Les valeurs acquises pour le rendement sur différents sites ont été traitées en utilisant les statistiques descriptives, à travers les calculs des valeurs moyennes, d'écarts types et de fréquences, avec le logiciel SPSS 12.0. Les données vérifiant les tests de normalité et d'homogénéité ont été soumises au test de Student-Newman-Keuls (Glèlè Kakai et al., 2004).

\section{RESULTATS}

Champignons identifiés dans les extraits de feuilles et effets des conservateurs sur la flore fongique. Les analyses microbiologiques ont montré une variabilité de la flore microbienne des extraits aqueux de feuilles (neem, papayer et hyptis) obtenus en utilisant les méthodes de pilage et d'extracteur motorisé.

Cas de l'extrait des feuilles de papayer non embouteillé. Plusieurs genres de champignons ont été identifiés au premier jour de l'extraction dans les extraits de papayer, quelle que soit la méthode d'extraction, les principaux genres de champignons dans les extraits des feuilles de papayer obtenus par pilage et par 1'extracteur sont Penicillium spp., Aspergillus spp., Fusarium spp., Nigrospora spp. et Stemphylium spp. (Fig. 1 a). Une baisse significative de la charge fongique a été notée de façon générale durant les quatre (4) jours de conservation. Cependant, la charge en Aspergillus spp. a augmenté au cours des 4 jours de conservation et cette tendance est plus importante au niveau de l'extrait issu de la méthode traditionnelle $\left(7,110^{3} \mathrm{CFU} \mathrm{mL}^{-1}\right.$ contre $210^{3} \mathrm{CFU} \mathrm{mL}^{-1}$ pour l'extrait issu de l'extracteur). Ces résultats montrent que les différents lots de feuilles utilisés pour l'expérimentation sont contaminés d'une part et d'autre part la flore fongique n'est pas homogène.

Cas de l'extrait des feuilles de papayer embouteillé. Les mêmes analyses ont été effectuées sur des échantillons d'extrait de papayer ayant reçu ou non des conservateurs et stockés pendant 1 et 2 mois. Aucune moisissure n'a été dénombrée dans les extraits (avec conservateurs ou non) après un (1) et deux (2) mois de conservation.

Cas de l'extrait des feuilles d'hyptis non embouteuillé. Les principaux genres de la

TABLEAU 1. Traitements appliqués aux cultures de niébé (extrait aqueux conservés pendant 45 jours et 6 mois)

\begin{tabular}{|c|c|c|c|c|c|c|}
\hline \multirow[t]{2}{*}{ Parcelles (P) } & \multicolumn{2}{|c|}{ Extrait de feuille de neem issu de : } & \multicolumn{4}{|c|}{ Conservateurs } \\
\hline & Mortier & $\begin{array}{l}\text { Extracteur } \\
\text { motorisé }\end{array}$ & Citron & Alcool & $\begin{array}{c}\text { Ocimum } \\
\text { basilicum }\end{array}$ & $\begin{array}{c}\text { Ocimum } \\
\text { graticimum }\end{array}$ \\
\hline 1 & $\mathrm{x}$ & & & & & \\
\hline 2 & & $\mathrm{x}$ & & & & \\
\hline 3 & $\mathrm{x}$ & & $\mathrm{x}$ & & & \\
\hline 4 & & $\mathrm{x}$ & $\mathrm{x}$ & & & \\
\hline 5 & $\mathrm{x}$ & & & $\mathrm{x}$ & & \\
\hline 6 & & $\mathrm{x}$ & & $\mathrm{x}$ & & \\
\hline 7 & $\mathrm{x}$ & & & & $\mathrm{x}$ & \\
\hline 8 & & $\mathrm{x}$ & & & $\mathrm{x}$ & \\
\hline 9 & $\mathrm{x}$ & & & & & $\mathrm{x}$ \\
\hline 10 & & $\mathrm{x}$ & & & & $\mathrm{x}$ \\
\hline
\end{tabular}



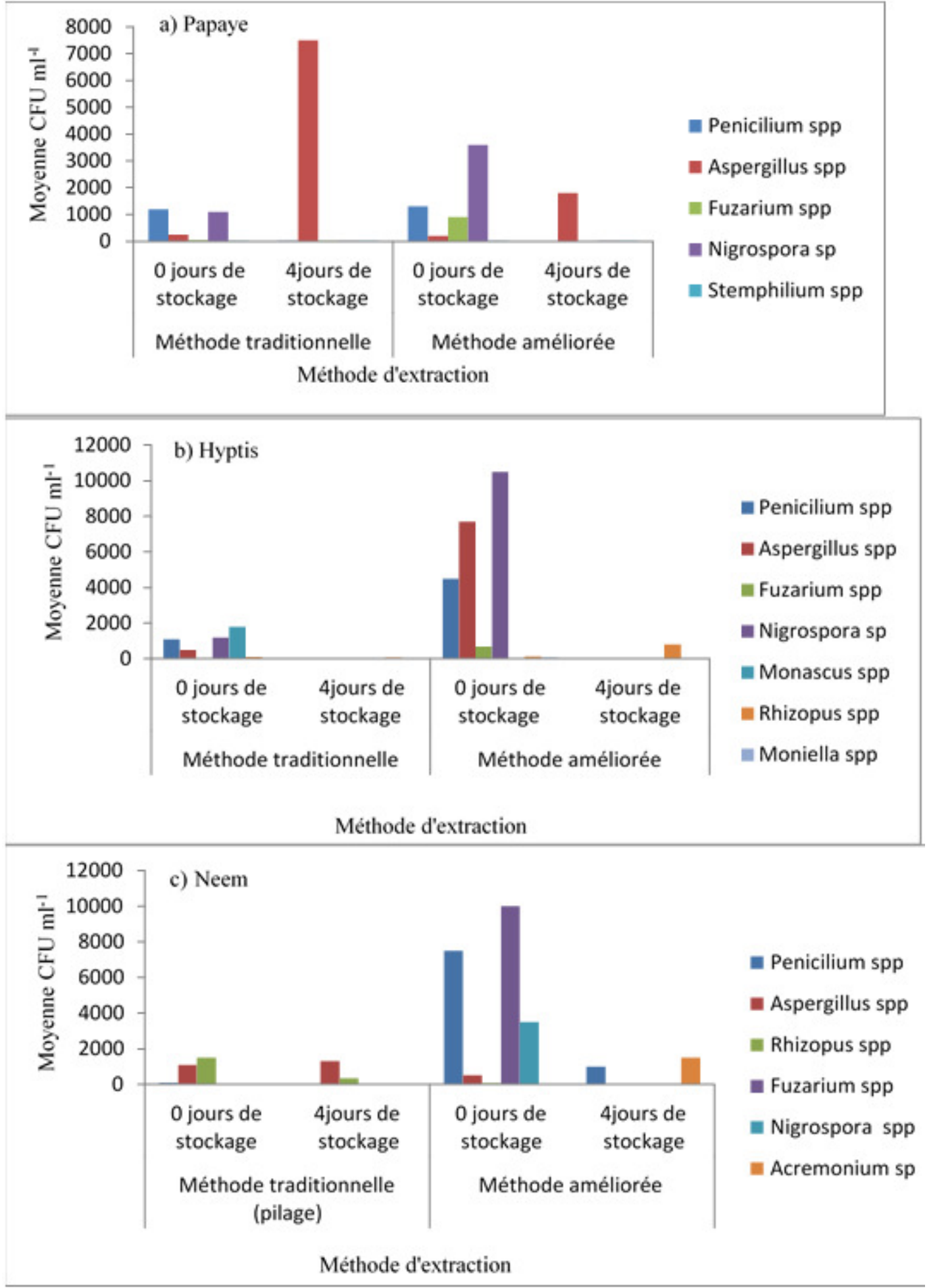

Figure 1. Charge fongique de l'extrait de feuilles de papayer, d' hyptis et de neem par méthode d'extraction et en fonction du temps de stockage. 
flore fongique dénombrés dans les extraits d'hyptis (Fig. 1 b) sont Penicillium spp., Aspergillus spp., Fusarium spp., Nigrospora spp., Monascus spp., Rhizopus spp., Moniella spp. Nigrospora spp. $\left(10^{4} \mathrm{CFU} \mathrm{ml}^{-1}\right)$ a été le genre le plus important au début dans l'extrait d'hyptis obtenu avec la méthode améliorée suivi $\mathrm{du}$ genre Aspergillus puis du genre Penicillium. Une diminution significative $(\mathrm{P}<0,01)$ a été observée au cours du stockage au niveau des deux méthodes, après 4 jours de stockage.

Cas de l'extrait des feuilles d'hyptis embouteuillé. Après 1 et 2 mois de stockage, la charge fongique des extraits avec conservateurs, a connu une décroissance significative. Seuls Penicillium spp. a été identifié dans les traitements hyptis pilage + Ocimum basilicum (10 CFU $\left.\mathrm{mL}^{-1}\right)$, hyptis

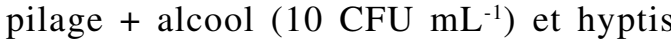
extracteur + jus de citron $\left(10^{2} \mathrm{CFU} \mathrm{mL}^{-1}\right)$. Rhizopus spp. a été identifié au niveau du traitement hyptis extracteur + Ocimum gratissimum. Après 2 mois de stockage, aucun genre de champignon n'a été identifié au niveau des différents traitements.

Cas de l'extrait de neem non embouteillé. Les principaux genres de champignons identifiés dans les extraits de feuilles de neem sont Aspergillus spp., Penicillium spp., Fusarium spp., Acremonium spp., Rhizopus spp., Nigrospora spp. (Fig. 1 c). En général, la charge fongique en $\mathrm{CFU} \mathrm{ml}{ }^{-1}$ décroît de 0 à 4 jours de stockage. En effet, pour la méthode traditionnelle (pilage), la charge en Rhizopus spp. a été de $10^{3} \mathrm{CFU} \mathrm{ml}{ }^{-1}$ juste après extraction et cette charge est devenue nulle après 4 jours de stockage. La même tendance a été observée au niveau des extraits obtenus avec l'extracteur motorisé. Pour le Fusarium spp, la charge a été de $10^{4} \mathrm{CFU} \mathrm{ml}{ }^{-1}$ juste après extraction et de $0 \mathrm{CFU} \mathrm{ml}{ }^{-1}$ après 4 jours de stockage (Fig. 1 c). Cette décroissance de la charge en Fusarium spp. a été significative pendant la durée de stockage quelle que soit la méthode d'extraction utilisée $(\mathrm{P}<0,01)$.

Cas de l'extrait de neem embouteillé. A un (1) mois de stockage, à l'exception de l'extrait de neem issus de l'extracteur sans ajout de conservateur, celui avec ajout de citron et de l'extrait issu du pilage avec ajout de d'Ocimum gratissimum, aucun genres initialement identifiés n'a été retrouvé dans les extraits de neem. En effet, après 1 mois de stockage, le genre Penicillium spp. a été retrouvé (10 CFU ml $\left.{ }^{-1}\right)$ dans l'extrait de neem pilé + Ocimum gratissimum. Par contre Penicillium spp. a été très nombreux au niveau du témoin (extrait sans conservateur) et de l'extrait de neem issu de l'extracteur + jus de citron. Après 2 mois de stockage, seuls Penicillium spp. (10 CFU ml $\left.{ }^{-1}\right)$ et Rhizopus spp. (10 CFU ml ${ }^{-1}$ ) ont été présents dans l'extrait de neem issu de l'extracteur et absents au niveau des deux autres traitements. L'huile essentielle d'Ocimum gratissimum semble avoir inhibé le développement et la prolifération des moisissures. Ce résultat montre que l'action inhibitrice de Ocimum gratissimum et celle du jus de citron qui ont été progressives et certainement persistante dans le temps. L'analyse statistique montre que la décroissance du nombre de colonies est significative dans le temps $(\mathrm{P}<0,01)$.

\section{Efficacité technique des extraits aqueux avant et après conservation}

Rendements du niébé traité avec les extraits aqueux du jour. La Tableau 2 montre que les rendements en grains des parcelles de niébé traitées avec les extraits aqueux du jour obtenus à partir des deux méthodes et des feuilles de neem, de papayer et d'hyptis varient de 1191,66 à $1481 \mathrm{~kg} \mathrm{ha}^{-1}$. L'introduction de l'extracteur pour la production des extraits aqueux n'a pas d'effets sur l'efficacité avérée des extraits aqueux pour le traitement du niébé. Les extraits aqueux du jour obtenus avec 
l'extracteur sont aussi efficaces que les extraits obtenus par la méthode traditionnelle de pilage.

Rendement du niébé traité avec des extraits aqueux de neem conservés pendant 45 jours et 6 mois. Les rendements de la variété Kplobè de niébé traitée avec l'extrait aqueux conservé pendant 45 jours et ceux de la variété Flore traitée avec l'extrait aqueux conservé pendant 6 mois sont présentés dans le Tableau 3. Les rendements obtenus avec les extraits aqueux avec ou sans conservateurs conservés pendant 45 jours varient entre $1219 \mathrm{~kg} \mathrm{ha}^{-1}$ et $1820 \mathrm{~kg} \mathrm{ha}^{-1}$. Ces rendements sont parfois plus élevés que ceux obtenus avec le produit de synthèse Fénithroton qui présente un rendement de $1456,67 \mathrm{~kg} \mathrm{ha}^{-1}$. Aussi, les rendements obtenus avec le neem sans conservateurs pilé ou extrait par l'extracteur sont supérieurs à ceux obtenus avec le témoin et de façon significative. Il en est de même avec le neem pilé et conservé avec l'Ocimum basilicum qui d'ailleurs a donné le meilleur résultat. Avec les autres traitements, la

TABLEAU 2. Rendements du niébé (Kg/ha) traités avec les extraits aqueux de neem du jour

\begin{tabular}{lll}
\hline Type de traitements & Mode d'obtentionde l'extrait & Rendement \\
\hline Fénithroton & (Témoin) & $1369,5 \pm 56$ (a) \\
Extrait aqueux de neem & Pilage & $1254,6 \pm 38$ (a) \\
Extrait aqueux de neem & Extracteur & $1255,3 \pm 30$ (a) \\
Extrait aqueux de papayer & Pilage & $1191,6 \pm 44$ (a) \\
Extrait aqueux de papayer & Extracteur & $1277 \pm 29$ (a) \\
Extrait aqueux d'hyptis & Pilage & $1481 \pm 17$ (a) \\
Extrait aqueux d'hyptis & Extracteur & $1336 \pm 52$ (a) \\
\hline
\end{tabular}

Les chiffres portant les mêmes lettres ne sont pas différents au seuil de 5\%

TABLEAU 3. Rendement du niébé traité avec des extraits aqueux de neem conservés pendant 45 jours (variété Klpobè) et 6 mois (variété Flore)

\begin{tabular}{lcc}
\hline Temps de conservation extraits & \multicolumn{2}{c}{ Rendement $\left(\mathrm{kg} \mathrm{ha}^{-1}\right)$} \\
\cline { 3 - 3 } & 45 jours & 6 mois \\
\hline Types de traitements & & \\
& & - \\
Fénithroton (témoin) & $1456,67 \pm 16(\mathrm{ab})$ & $600,00 \pm 13(\mathrm{ab})$ \\
Neem du jour (pilage; témoin) & $1598,67 \pm 89(\mathrm{bc})$ & $620,00 \pm 78(\mathrm{ab})$ \\
Neem du jour (extracteur; témoin) & $1646,67 \pm 10(\mathrm{bc})$ & $362,50 \pm 11(\mathrm{a})$ \\
Neem sans conservateur (pilage) & $1580,00 \pm 00(\mathrm{bc})$ & $437,50 \pm 55(\mathrm{a})$ \\
Neem sans conservateur (extracteur) & $1531,00 \pm 00(\mathrm{abc})$ & $675,00 \pm 16(\mathrm{ab})$ \\
Neem + Citron (pilage) & $1510,00 \pm 00(\mathrm{abc})$ & $575,00 \pm 14(\mathrm{ab})$ \\
Neem + Citron (extracteur) & $1219,00 \pm 00(\mathrm{a})$ & $550,00 \pm 16(\mathrm{ab})$ \\
Neem + Alcool (pilage) & $1820,00 \pm 00(\mathrm{~d})$ & $737,50 \pm 16(\mathrm{ab})$ \\
Neem + Alcool (extracteur) & $1376,00 \pm 00(\mathrm{ab})$ & $837,50 \pm 14(\mathrm{ab})$ \\
Neem + Ocimum basilicum (extracteur) & $1440,00 \pm 00(\mathrm{ab})$ & $572,50 \pm 12(\mathrm{ab})$ \\
Neem + Ocimum basilicum (pilage) & $1532,00 \pm 00(\mathrm{abc})$ & $587,50 \pm 14(\mathrm{ab})$ \\
Neem + Ocimum graticimum (pilage) & $1598,67 \pm 89(\mathrm{bc})$ & $812,50 \pm 76(\mathrm{ab})$ \\
Neem + Ocimum graticimum (extracteur) & $1532,00 \pm 00(\mathrm{abc})$ &
\end{tabular}

Les chiffres portant les mêmes lettres dans la même colonne sont statistiquement identiques 
différence avec le témoin n 'est pas significative au seuil de $5 \%$.

En ce qui concerne les rendements de niébé variété Flore traités avec les extraits de neem consommés pendant 6 mois, ils sont globalement faibles indépendamment du type de traitement. Les rendements varient de 362,5 $\mathrm{kg} \mathrm{ha}^{-1}$ à $837,5 \mathrm{~kg} \mathrm{ha}^{-1}$. Les extraits aqueux conservés sans ajout de conservateurs ont les plus faibles rendements tandis que ceux conservés avec les huiles essentielles ont les meilleurs rendements en niébé (Tableau 3). Les rendements du niébé traité avec les extraits aqueux conservés avec les huiles essentielles, l'alcool et le jus de citron ne sont pas statistiquement différents $(\mathrm{P}>0,05)$ de ceux obtenus sur les parcelles de niébé traitées avec les extraits aqueux de neem du jour.

\section{DISCUSSION}

La diminution significative de la flore fongique des extraits de feuilles sans conservateurs observée au cours du stockage montre que les extraits aqueux des feuilles comportent probablement des substances actives qui auraient inhibé le développement et la prolifération des moisissures (Biswas et al., 2002). Ces substances ont donc des propriétés antifongiques. En effet, l'extrait d'hyptis a une activité antifongique très prononcée sur les moisissures en particuliers les espèces Aspergillus flavus, Aspergillus parasiticus, Asperillus ochraceus, Fusarium oxysporum (Adjou et Soumanou, 2013). Ces différents genres de moisissures (Aspergillus spp. et Fusarium spp.) ont été également dénombrés dans les extraits aqueux étudiés avant conservation. Les différents extraits aqueux des feuilles étudiés contiennent des composés actifs antimicrobiens qui leur permettent de tuer ou ralentir la croissance des microorganismes (Agban et al., 2017 (hyptis) ; Tamokou et al. 2017 (papaye); Hala et Al Fadhil. 2015 (neem)). Par ailleurs, la plupart des genres recensés sont aérobies (Amani, 2016). En conséquence, le conditionnement des extraits dans les bocaux fermés hermétiquement pourrait empêcher le développement et la prolifération des moisissures par manque d'oxygène. Une variabilité a été notée au niveau de la flore fongique contaminant les extraits suivant le type de feuille. Les feuilles seraient donc contaminées par les spores de champignons se trouvant dans le sol et dans l'air avant extraction. Un lavage des feuilles avant extraction permettrait la réduction de ces spores avant extraction.

Après 1 et 2 mois de conservation, la plupart des genres de moisissures recensés au début ont été réduits voire absents. Cette réduction serait due d'une part, à la nature aérobie des moisissures identifiées qui ne favorise pas leur développement dans les bouteilles et d'autre part, à la présence de substances actives dans les extraits qui ont des effets antifongiques. C'est le cas des feuilles d'hyptis qui contiennent des phénols ayant des propriétés antifongiques (Edega et al., 2006). Les huiles essentielles d'Ocimum graticimum et d'Ocimum basilicum contiennent aussi des phénols et autres composés phénoliques qui ont des propriétés antimicrobiennes et antifongiques (Fandohan et al., 2004 ; Edega et al., 2006). L'utilisation des conservateurs a certainement eu un effet inhibiteur sur les moisissures. Les conservateurs tels que Ocimum basilicum et Ocimum gratissimum semblent contrôler significativement la flore fongique. Ce résultat est en concordance avec les résultats des travaux antérieurs qui ont prouvé l'effet antifongique de ces huiles essentielles sur les champignons des stocks (Owolade et al., 2000; Soliman and Badeaa, 2002; Fandohan et al., 2004) et plus spécifiquement sur les genres Aspergillus spp et Fusarium spp (Adjou et Soumanou, 2013). Cependant, le genre Penicillium spp semble être moins sensible aux effets des extraits de feuilles de neem et de l'hyptis et des conservateurs pendant le $1^{\text {er }}$ mois de conservation. Quant au citron, il a un effet inhibiteur après 2 mois de stockage sur tous 
les genres identifiés. Le citron a certainement un effet progressif et persistant alors que les huiles essentielles d'Ocimum basilicum et Ocimum gratissimum et alcool $\left(70^{\circ} \mathrm{C}\right)$ semblent avoir des effets immédiats et persistants.

D’une façon générale, la diminution de la flore fongique pourrait être aussi due à une synergie des effets antifongiques notamment des composés actifs des extraits et des conservateurs à laquelle s 'ajoute le fait que les extraits, une fois embouteillés, les germes se retrouvent dans des conditions anaérobiques qui défavorisent leur développement. L'utilisation des huiles essentielles d'Ocimum basilicum et d'Ocimum gratissimum, du citron ou de l'alcool comme conservateurs pourrait être alors recommandée.

En ce qui concerne, les rendements de niébé traité avec les extraits conservés, une efficacité des extraits aqueux conservés pendant 45 jours a été notée. Indépendamment de la méthode d'extraction, les extraits aqueux ont conservé leur principe actif après 45 jours de conservation. Les valeurs du rendement en niébé sont proches de celles obtenues par Gbaguidi et al. (2015) qui rapportent une valeur de $1679 \mathrm{~kg} / \mathrm{ha}$ obtenue en station avec la variété Kplobè. Quant aux rendements du niébé traité avec les extraits de neem conservés pendant 6 mois, ils sont relativement faibles. Ceci est certainement dû aux poches de sécheresse et à la faible pluviométrie qui a sévit dans la localité (commune d'Adjohoun ) pendant la période des essais. Toutefois, on note une bonne performance des extraits conservés avec les conservateurs par rapports à l'extrait aqueux du jour utilisé comme témoin. Les extraits aqueux conservés sans ajout de conservateurs ont été moins efficaces que les extraits aqueux du jour et ceux conservés avec des conservateurs. Les rendements obtenus avec les extraits aqueux stockés sans conservateurs sont environ deux (2) fois plus faibles que ceux obtenus avec les autres extraits (du jour et ceux conservés avec conservateurs). On pourrait en déduire que la conservation des extraits aqueux avec des conservateurs tels que le jus de citron, l'alcool, les huiles essentielles Ocimum graticimum et Ocimum basilicum permet de ralentir la perte du principe actif de ces extraits aqueux. Une confirmation de ces résultats sur une deuxième année savère nécessaire afin de tirer des conclusions définitives.

\section{CONCLUSION}

Indépendamment de la feuille, les extraits aqueux du jour obtenus avec l'extracteur sont aussi efficaces sur les cultures de niébé que les extraits obtenus par la méthode traditionnelle de pilage. L'utilisation des conservateurs a eu un effet inhibiteur sur la flore fongique des extraits. Les rendements du niébé traité avec les extraits aqueux conservés avec les huiles essentielles, l'alcool et le jus de citron ne sont pas statistiquement différents à ceux obtenus sur les parcelles de niébé traitées avec les extraits aqueux de neem du jour. La conservation des extraits aqueux n'affecte donc pas leur efficacité. Les tests d'efficacité des extraits aqueux conservés pendant plus d'un an sont nécessaires pour consolider les résultats obtenus. A moyen terme, l'introduction de cette technologie permettra d'améliorer le taux d'adoption des extraits à base des plantes tels que le neem, l'hyptis et le papayer, toute chose qui réduirait l'effet néfaste de l'utilisation des pesticides de synthèse sur la santé des producteurs et sur l'environnement.

\section{REMERCIEMENT}

Les auteurs tiennent à remercier les producteurs de niébé des communes d'Aplahoué, de Klouekanme et d'Adjohoun pour avoir participé à l'étude. Nos remerciements vont également à l'endroit de la Coopération danoise (DANIDA) pour avoir financé cette étude. 


\section{REFERENCES BIBLIOGRAPHIQUES}

Adéoti, R., Coulibaly, O. and Tamo, M. 2002. Facteurs affectant l'adoption des nouvelles technologies de niébé Vigna unguiculata en Afrique de l'Ouest. Bulletin de la Recherche Agronomique du Benin $\mathrm{n}^{\circ} 36.18 \mathrm{pp}$.

Adjou, S.E. et Soumanou M.M. 2013. Efficacité des extraits de plantes dans la lutte contre les moisissures toxinogènes isolées de l'arachide en post-récolte au Bénin. Journal of Applied Biosciences 70: 5555-5566. ISSN 1997-5902

Affokpon, A., Djihinto, C.A., ZandjanakouTachin, M., Tossou, C., Achigan dako, E.G., Baimey, H.K. et Bokonon-Ganta, A.H. 2017. Gestion des nematodes a galles parasites de la culture d'egusi par l'utilisation de Trichoderma asperellum et de la poudre de graine de neem. African Crop Science Journal 25(2):133-143.

Agban, A., Atchou, K., Tchacondo, T., Hoekou, Y. P., Batawila, K. et De Souza, C. 2013. Potentiel antimicrobien des extraits de feuilles d'Hyptis suaveolens poit. Journal Recherches Sciences. Universitaire. Lomé (Togo), Série A 15(3):37-44.

Ahouansou, R.H., Agbobatinkpo, P., Agli, C., Dededji Montcho, M., Adekambi, S. et Aly, D. 2014. Etude technique et économique de la production conservation des extraits aqueux de feuilles de neem au Bénin. Spécial hors série ${ }^{\circ} 1$, Mai 2014. Science et Technique, Lettres, Sciences Sociales et Humaines 1: 85-99.

Ahmed, B.I., Abdulhameed, A., Yusuf, S.R. and Aliyu, M. 2006. Comparative study of the defoliator activities of Podagrica sjostedti and P. uniforma (Coleoptera: Chrysomelidae) on two intercropped Okra varieties in Bauchi State, Nigeria. Savannah Journal of Agriculture 12:12-14.

Ahmed, B.I., Onu, I. and Mudi, L. 2009. Field bioefficacy of plant extracts for the control of post flowering insect pests of cowpea
(Vigna unguiculata (L.) Walp.) in Nigeria. Journal of Biopesticides 2(1):37-43.

Akakpo, C. et Allagbé, M. 2002. Contrôle du complexe parasitiaire du niébé avec l'extrait aqueux de feuilles de hyptis à Zouzouvou et Eglimé. Actes de l'Atelier Scientifique Sud-Centre, Niaouli 11-12 Décembre 2002. pp. 126-129.

Amani, L. 2016. Mycotoxines et champignons mycotoxinogènes dans les grains de sorgho commercialisé en Tunisie : Incidence et profils écophysiologiques. Thèse de Doctorat. Institut Supérieur de Biotechnologie de Monastir. 225pp.

Atropo, K., Yehouénou, A. et Komlan, F. 2001. Utilisation des extraits aqueux de feuille de neem pour la protection des légumes feuilles. Actes de l'Atelier Scientifique SudCentre, Niaouli 12-13 Décembre 2001. pp. 78-84.

Belanger, A. et Musabyimana, T. 2010. Le Neem contre les insectes et les maladies. Agriculture et Agroalimentaire Canada, Centre de Recherche et de Développement en Horticulture. ASPRO. 13pp.

Biswas, K., Chattopadhyay, I., Banerjee, R. and Bandyopadhyay, U. 2002. Biological activities and medicinal properties of neem (Azadirachta indica). Current Science 82: 1336-1345.

Consultative Group on International Agricultural Research (CGIAR). 2011. Cowpea (Vigna unguiculata). Online Available: <http://www.cgiar.org/impact/ research>/cowpea.html [Accessed on 23 April, 2011].

Dzemo, W.D., Niba, A.S. and Asiwe, J. 2010. A comparative study of the bionomics of Clavigralla tomentosicollis Stäl (Hemiptera: Coreidae) on tree varieties of cowpea (Vigna unguiculata L. Walp). African Journal of Agricultural Research 5(7): 567-572. DOI: 10.5897/AJAR09.724. ISSN 1991-637X @) 2010 Academic Journals 
Egho, E.O. and Ilondu, E.M. 2012. Seeds of neem tree (Azadirachta indica A. Juss). promising biopesticide in the management of cowpea insect pests and grain yield in the early cropping season at Asaba and Abraka, Delta State, Nigeria. Journal of Agricultural Science 4(1):181-189 doi:10.5539/jas.v4n1p181

Fandohan, P., Gbenou, J.D., Gnonlonfin, B., Hell, K., Marasas W.F.O. and Wingfield, M.J. 2004. Effect of essential oils on the growth of Fusarium verticillioides and fumonisins contamination in corn. Journal of. Agriculture and Food Chemistry 52: 6824-6829.

FAOSTAT. 2010. Agricultural production, crop primary database. Food and Agricultural Organization of the United Nations, Rome. http://faostat.fao.org/faostat

Gbaguidi, A.A., Assogba, P., Dansi, M., Yedomonhan, H. and Dansi, A. 2015. Caractérisation agro morphologique des variétés de niébé cultivées au Bénin; International Journal of Biological and Chemical Science 9(2):1050-1066 ; ISSN 1997-342X (Online), ISSN 1991-8631 (Print)

Glèlè Kakaï, R. et Kokode, G. 2004. Techniques statistiques univariées et multivariées: applications sur ordinateur. Bibliothèque Nationale, Bénin.

Giglioti, R., Forimb, M.R., Oliveira, H.N., Chagasc, A.C.S., Ferrezinid, J., Britoe, L.G., Falcoskif, T.O.R.S., Albuquerquea, L.G. and Oliveirac, M.C.S. 2011. In vitro acaricidal activity of neem (Azadirachta indica) seed extracts with known azadirachtin concentrations against Rhipicephalus microplus; Veterinary Parasitology 181:309 - 315; DOI:10.1016/ j.vetpar.2011.03.053

Grebmer, V., Bernstein, K.I., Nabarro, J., Prasai, D., Amin, N., Yahannes, S., Yisehac, Sonntag, Patterson, A., Towey, F., Thompson, O. and Jennifer. 2016. Indice de la faim dans le monde, Edition
Afrique. NEPAD, RESAKSS, Welt Hunger Hilfe, IFPRI.

Hafiz, N.A. and Damarany, A.M. 2006. Variation in the susceptibility of some cowpea (Vigna unguiculata (L.) Walp.) genotypes to infestation with certain pest in Egypt. Assuit University Bulletin Environmental Researches 9(1):7-15.

Houndété, T., Arodokoun, D. and Ahohouendo, B. 2002. Conservation de la solution d'extraits aqueux de feuilles de neem: Stabilisation au jus de citron. Actes de l'Atelier Scientifique Centre, Dassa 18-19 Décembre 2002. pp. 84-92.

Kadri, A., Zakari Moussa, O., Sido Yacouba, A., Hame Abdou, K.K. et Karimoune, L. 2013. Gestion intégrée de Maruca vitrata (FABRICIUS, 1787) et Megalurothrips sjostedti (TRYBOM, 1908), deux insectes ravageurs majeurs du niébé au Niger. International Journal of Biological and Chemical Science 7(6):2549-2557.

Kwaifa, N.M., Ibrahim, B.I., Aliyu, U.A., Muhammad and Dangana, A. 2015. Insecticidal effects of neem kernel extracts on flea beetle (Podagrica uniforma J.) of okra (Abelmoschus esculentus L.) in Jega, Kebbi, Nigeria. IOSR Journal of Agriculture and Veterinary Science 8(12): 57-60. DOI: 10.9790/2380-081225760.

Mamuda, M., Daudab, M. and Bongfac, B. 2016. Influence of formulated neem seed oil and Jatropha curcas seed oil on wire drawing of mild steel and medium carbon steel at elevated temperatures. Journal Tribology 10:16-27.

Makanur, B., Deshpande, V.K. and Vyakaranahal, B.S. 2013. Characterization of cowpea genotypes based on quantitative descriptors. Academic Journals 8(4):11831188.

Mukendi, R., Tshlenge, P., Kabwe, C. and Munyuli, T.B.M. 2014. Efficacité des plantes médicinales dans la lutte contre ootheca mutabilis sahlb. (chrysomelidae) en champ de niébé (Vigna unguiculata (L.) 
Walp.) en RD du Congo. Lebanese Science Journal 15(1):

Munyuli, T.M.B., Kyamanywa, S. and Luther, G.C. 2008a. Capability of Forficula auricularia L. (Dermaptera: Forficulidae) to prey on Aphis craccivora K.(Homoptera: Aphididae) in eastern and central Africa. Agronomie Africaine 20(1):57-66.

Ouali-N'goran, S.W.M., Boga, J.P., Johnson, F., Tano, Y. and Fouabi, K. 2014. Influence of dietary factors of five varieties of beans sold in Côte d'Ivoire on some biological parameters of Callosobruchus maculatus (Fab.) Coleoptera, Bruchidae. Journal of Animal \& Plant Sciences 21(1):3251-3262.

Owolade, O.F., Amusa, A.N. and Osikanlu, Y.O.K. 2000. Efficacy of certain indigenous plants extracts against seedborne infection of Fusarium moniliforme on maize (Zea mays L.) in South Western Nigeria. Cereal Research Communications 28:323-327.

Pazinato, R., Volpato, A., Baldissera, M.D., Santos, R.C.V., Baretta, D., Vaucher, R.A., Giongo, J.L., Boligon, A.A., Stefani, L.M. and Da Silva, A.S. 2016. In vitro effect of seven essential oils on the reproduction of the cattle tick Rhipicephalus microplus. Journal of Advanced Research 7:10291034. http://dx.doi.org/10.1016/j.jare. 2016.05.003

Shanko, D., Andargie, M. and Zelleke, H. 2014. Interrelationship and path coefficient analysis of some growth and yield characteristics in cowpea (Vigna unguiculata L. Walp) Genotypes. Journal of Plant Sciences 2(2):97-101. Doi: 10.11648/j.jps.20140202.13

Soliman, K.M. and Badeaa, R.I. 2002. Effect of oil extracted from some medicinal plants on different mycotoxigenic fungi. Food Chem. Toxicol. 40:1669-1675. https:// doi.org/10.1016/S0278-6915(02)00120-5

Tamgno, B.R. et Ngamo Tinkeu, S.L. 2014. Utilisation des produits dérivés du neem Azadirachta indica A. Juss comme alternatifs aux insecticides synthétiques pour la protection des semences de maïs et de sorgho dans la Vallée du Logone. Sciences, Technologies et Développement 15:1-8.

Tamokou, J., Ekom, S.E., Njouendou, A.J., Fosso, A.J.M. and Kengne, I.C. 2017. Antibacterial activities of methanol extracts of Carica papaya, Ocimum gratissimum and Solanum torvum under normal and osmotic stress conditions. Journal of Complementary and Alternative Medical Research 4(2):1-11. ISSN: 2456-6276

Zoffoun, A., Awaka, A., Akakpo, C. et Allagbé, M. 2004. Contrôle du complexe parasitaire du niébé avec l'extrait aqueux d'hyptis. Actes de l'Atelier Scientifique Sud (4 ème edition) Abomey-Calavi ; 14-17 Décembre 2004. pp. 21-26. 